\title{
Ultrasound-Guided Central Venous Catheterization: A Protocol to Be Followed in Pediatrics?
}

\section{Mayara Gonçalves Marques, Regina Grigolli Cesar}

Pediatrics Department-Pediatric Intensive Care Unit, Irmandade da Santa Casa de Misericórdia de São Paulo, São Paulo, Brazil Email: reginautiped@uol.com.br

How to cite this paper: Marques, M.G. and Cesar, R.G. (2017) Ultrasound-Guided Central Venous Catheterization: A Protocol to Be Followed in Pediatrics? Open Journal of Pediatrics, 7, 128-139.

https://doi.org/10.4236/ojped.2017.73016

Received: June 27, 2017

Accepted: August 1, 2017

Published: August 4, 2017

Copyright $\odot 2017$ by authors and Scientific Research Publishing Inc. This work is licensed under the Creative Commons Attribution International License (CC BY 4.0).

http://creativecommons.org/licenses/by/4.0/

\begin{abstract}
Objective: To investigate the effectiveness of ultrasound-guided central venous catheterization when compared to the conventional procedure. Method: A prospective cohort study was carried out over a 9-month period from February to October 2016 involving 144 inpatients at PICU of Irmandade da Santa Casa de São Paulo Hospital, undergoing central venous catheterization. The patients were matched in pairs of identical patients according to the levels of potentially intervening variables (age, nutritional status, puncture site, professional experience), differing only as to the CVC technique: ultrasound-guided (USG-CVC) or conventional (C-CVC). Discarding data from non-paired patients, the remaining did forming 47 pairs, matched as two related samples: USG-CVC and C-CVC groups. Success parameters: number of puncture attempts; time spent at CVC; success rate and complications. Results: In the USG-CVC group, the number of attempts (mean $=2.04$ ) and the time spent at catheterization (mean $=11.89$ minutes) were lower $\left(t=2.34, \mathrm{df}=46, t_{0.95}=\right.$ 2.02, $p<0.05)$ and $\left(t=3.07, \mathrm{df}=46, t_{0.95}=2.02, p<0.05\right)$, respectively, when compared to the results obtained for the control group (C-GVC), (mean = 3.21 ) and (mean $=28.26$ minutes), respectively. As to success, there was observed a significant difference $\left(F_{(1,46)}=16.6 ; Q_{(1)}=12.5, p<005\right)$ when considering only one trial (USG -CVC $=27 / 47$; C-CVC $=9 / 47$ ), but no significant difference $\left(F_{(1,46)}=3.76 ; Q_{(1)}=3.56, p>0.05\right)$ when considering several attempts. Complications were found less frequently in the USG-CVC group $(3 / 47)$ than in the CVC-C (13/47), $\left(F_{(1,46)}=8.24 ; Q_{(1)}=7.14, p<0.05\right)$. Conclusion: USG-CVC was found to be more effective than the conventional technique, especially regarding success at the first puncture attempt.
\end{abstract}

\section{Keywords}

Central Venous Catheters, Ultrasonography, Central Venous Catheterization, Pediatrics, Pediatric Intensive Care Units, Patient Safety 


\section{Introduction}

Central venous catheterization (CVC) is defined as the placement of an appropriate vascular access device whose extremity reaches the superior or inferior vena cava irrespective of the peripheral insertion site [1]. The procedure, used since the 1930s and widespread in Pediatrics from 1945 [2], has become an important part of the clinical management in patients admitted to the pediatric intensive care unit (ICU).

The intravascular access procedure can be performed by percutaneous puncture or surgical dissection of the vessel to be catheterized, and the catheter can be placed into several venous sites such as jugular, subclavian, and femoral sites. There are some descriptions in the literature of percutaneous puncture of axillary veins [3]; however, this route has not been used routinely in most services.

When choosing the site, the clinical status of the patient, the experience of the practitioner and the indication for the insertion are to be considered. Sites are often chosen taking into account ease of insertion, reasons for use, and lower risk of complications. There is controversy as to the best access route. From comparing the jugular and the subclavian veins, there is evidence that more inadvertent arterial punctures occur, but few catheters remain poorly placed when the access is through the internal jugular vein [4]. Other authors have observed that complications from catheter insertion in the subclavian vein are more common [5].

In cases of puncture of the jugular vein or the subclavian vein, preference is given to the right side, since the pleural dome is lower (lower risk of pneumothorax, especially in subclavian puncture), the path to the right atrium is more rectilinear (lower possibility of misplacing the catheter, especially via the jugular), and the thoracic duct empties into the left subclavian vein (lower risk of chylothorax) [1].

CVC is neither a complication-free nor risk-free procedure. In pediatric ICUs, this procedure may be even more complex, given that it deals with a population with small venous calibers.

Paiva et al. showed in a study carried out in 2006 that among the complications stemming from catheter insertion in children, the most frequent ones were: catheter misplacement (17.6\%), arterial puncture (10.9\%), and pneumothorax (4.1\%). More than three puncture attempts were associated with complications ( $p=0.004)$. As to management-related complications, local infection was the main one $(68.2 \%)$, which in turn was associated with: catheter replacement with a guide wire $(p=0.008)$, use of mechanical ventilation $(p=0.025)$, and catheter in place for more than seven days $(p=0.001)$ [6].

In order to reduce the risks associated with central venous catheter insertion, the use of USG as a guide in the catheterization of the internal jugular vein, first described by Ullman and Stoelting in 1978 [7], has increased in recent years. The increasing number of publications has demonstrated its importance as a complementary tool in pediatric critical care, reducing the incidence of complica- 
tions and errors during catheter insertion.

Available in our practice, USG-guided CVC offers additional advantages, such as the absence of radioactive exposure for our patients, its practicality and portability, besides the fact that it is also a non-invasive method [8] [9].

Based on data obtained in several studies published from 1993 to 2000, later cited in a meta-analysis involving adults and infants by Hind et al., in 2001 [10], the US Agency for Healthcare Research and Quality already recommended the dynamic, real-time use of USG to guide catheterization aiming to increase patient safety [11]. In 2002, the UK's National Institute for Clinical Excellence recommended the use of a two-dimensional USG device in elective procedures involving venous catheter insertion into the internal jugular vein in adults and children [12]. In 2013, the US Agency for Healthcare Research and Quality reiterated its recommendation for using USG-CVC, thereby placing it among the 11 main safety practices aimed at improving patient care [13].

Studies in adults have shown that USG-guided central venous catheterization (CVC) reduces the number of attempts and time to successful vein catheterization [14].

The internal jugular vein is usually located anterolaterally to the carotid artery. Denys and Urestsky (1991) analyzed the position of the internal jugular vein in 200 adult patients. In $5.5 \%$ of the patients studied, the location of the internal jugular vein was shown to be off the position predicted by the conventional technique; in $8.5 \%$ of patients, the anatomy was sufficiently aberrant to complicate blind access [15]. Thus, the main advantage of the USG-CVC technique is the visualization of structures at the puncture site and the identification of inappropriate conditions for puncture such as thrombosis or fine caliber, as well as anatomical variations in the position of the vein in relation to the artery before the catheterization is attempted, thereby increasing the safety of the procedure [16] [17].

Unfortunately, there have been few studies to date involving the pediatric population, but there is sufficient evidence supporting the fact that the anatomy of cervical vessels in children is variable [18] [19]. The few studies that have been published on the vascular anatomy of infants and children have shown that the internal jugular veins may be located anteriorly, anterolaterally or posterolaterally to the artery.

Carotid arteries located posteriorly to the internal jugular vein are more prone to puncturing during CVC, being found with varying frequency according to the literature, apparently depending on the age group and as much as $10 \%$ in pediatric patients [20] and 54\% in adults [21].

Among the anomalies described by Alderson et al. (1993) in their ultrasound assessment of internal jugular veins, observed in $18 \%$ of the 50 children under the age of six included in the study, a very small diameter $(<3 \mathrm{~mm}$ in neonates and infants and $<5 \mathrm{~mm}$ in older children) was found in $4 \%$ of the internal jugular veins normally located. In $2 \%$ of cases, these veins were not visualized [20]. 
In 2014, Souza Neto et al. prospectively investigated the anatomical location among the internal jugular, subclavian and femoral veins of 142 pediatric patients, finding variations such as the internal jugular vein occupying a position that is lateral $(9 / 142)$ or anterior $(9 / 142)$ to the carotid artery, anteromedial to the femoral artery (17/142), and medial to the subclavian artery (10/142 patients) [22].

Over the last two decades, two CVC techniques on pediatric patients, namely the conventional and the ultrasound-guided techniques, have been compared as to the number of CVC attempts, time spent at catheterization, success rate, and complication rate during the procedure, such as arterial perforation. Verghese et al. (2000) also compared continuous wave Doppler USG-guided CVC coupled to a puncture needle, but this alternative technique proved to be less effective than the usual ultrasound technique [23].

As to the number of attempts, Verghese et al. (2000) found no difference in the number of arterial punctures when comparing both techniques [23]; nevertheless, the same authors reported, in 1999, a smaller number of attempts when CVC was guided by USG [24], which was to be later confirmed by Froelich et al. (2009) [25]. Jijeh et al. (2014) were successful at the first attempt in $75 \%$ of USG-guided punctures (mean time 3.9 minutes), 23\% at the second one, and 2\% at the third one, with no complications [26]. Pirotte \& Veyckemans (2007) had achieved an $84 \%$ success rate at the first attempt and $100 \%$ at the second one when performing dynamic USG-guided supraclavicular CVC [27].

With regard to the time spent, as found by Verghese et al. (1999), the use of USG reduced the time spent to catheter placement [24]. Pérez-Quevedo et al. (2016), in turn, the mean time to correct catheterization was 35.8 seconds [28]. Froelich et al. (2009) found no difference as to time, but commented that it varied also according to the practitioners' experience [25]. Grebenik et al. (2004) cite lack of experience as a limiting factor to the study [29].

With respect to the success rate, while in some studies [23] [25] [30] no statistically significant differences were found between the two CVC techniques, Verghese et al. (1999) and Grebenik et al. (2004) obtained higher success rates when using USG-guided CVC [24] [29], as well as other authors, who achieved $95 \%$ [26] and 96\% [28] success rates.

With regard to the occurrence of complications during CVC, Hind et al. (2003) reported that USG-guided CVC was safer [10]. Verghese et al. (1999), Grebenik et al. (2004) and Froelich et al. (2009) obtained a lower index of arterial punctures [24] [25] [29]. Alderson et al. (1993), in analyzing complications during internal jugular CVC related to anatomical variations of the internal jugular veins, reported a decrease from $60 \%$ to $20 \%$ when the procedure was guided by USG [20]. Pérez-Quevedo et al. (2016), found vascular perforation (11.9\%) to be the most frequent complication [28], whereas Iwashima, Ishikawa and Ohzeki (2008) found inadvertent puncture of the femoral artery as the most frequent complication in the group undergoing the conventional technique (32\% 
versus 7\% in the USG-assisted group) [30].

Based on the fact that both the size and weight of newborns and infants, as well as their greater mobility and the more superficial position and smaller diameter of some of their vascular structures may all contribute to greater technical difficulties, Pérez-Quevedo et al. (2016) recognize the need for training professionals under conditions simulating USG-guided CVC in pediatric patients and seek to obtain data that confirm that training on experimental models can reduce vascular catheterization complication rates in critically ill pediatric patients [28].

Aiming to obtain data to support the training of professionals in the USC-guided CVC technique, recently adopted but still underutilized in the pediatric ICU at Santa Casa de São Paulo, and its routine use for the population served, the present study was conducted to compare the USG-guided CVC with the conventional technique as to its effectiveness and occurrence of complications.

\section{Methodology}

\subsection{Patients}

The project was approved by the Institution's Research Ethics Committee.

This is a prospective cohort study where all children and adolescents (from one day to 18 years of age) admitted to the Pediatric Intensive Care Unit at Irmandade da Santa Casa de Misericórdia de São Paulo from February to October 2016 and undergoing central venous puncture in the unit's settings, and whose parents agreed to participate in the study, were studied.

\subsection{Design}

Considering the particularities of the service, including the recent introduction of the USG-guided CVC technique (which is not yet part of the routine), as well as the absence of electronic recording of medical data and (until recently) the absence of a logging protocol for data relative to this procedure, it was decided for a prospective follow-up and registration of patient data and protocol implementation in accordance with the US Agency for Healthcare Research and Quality guidelines (2001) [11].

The "CVC technique type" used (independent variable) at its two levels, namely conventional and USG-guide, will have its effectiveness assessed as to its possible effect on the outcomes "effectiveness parameters" and "occurrence of complications associated with the procedure" (dependent variables). Based on the literature, considered as effectiveness parameters were the catheterization success rate, the time spent to catheter placement, and the number of catheterization attempts.

Considering that the study was conducted at a teaching institution, most CVCs were carried out by relatively inexperienced ultrasound-guided CVC (USG-CVC) technicians, resident physicians specializing in pediatric intensive care in their 
third and fourth year, supervised by an experienced professor (preceptor). Occasionally, the procedure is carried out solely by the preceptor. In the present study the level of experience was considered a potentially competing variable over the independent variable-dependent variable effect (IV-DVs).

Other potentially intervening variables also considered were age group and nutritional status, in terms of Body Mass Index (BMI), as the possible effects on determining the anatomical reference points of the puncture sites, as well as the site chosen in each case.

In order to minimize the effect of the potentially intervening variables, we intended, at first, to treat the obtained data as coming from pairs of patients who were as similar as possible concerning the different levels of these variables, exposed (each element of a pair) to one of the two IV levels (USG-CVC; conventional CVC, C-CVC), in an attempt to counterbalance these effects. However, the frequent unavailability of the USG equipment at the pediatric ICU jeopardized the deliberately random exposure of patients to one of the two CVC techniques so as to obtain a true Matched Pair Design, with the choice being limited to the occasional availability of the equipment. We opted for a post hoc pairing.

\subsection{Procedure}

Hemodynamically stable patients, referred for CVC after having been suitably classified as to the levels of the potentially intervening variable Age group (newborn: 0 - 30 days of age; infant: 1 month to 2 years; preschool: 2 to 6 years; child: 6 to 12 years; adolescent: 12 to 18 years), Nutritional status (normal: 18.49 25.00; underweight; overweight), and Puncture site according to clinical indication (jugular; femoral; subclavian) were sedated with $0.2 \mathrm{mg} / \mathrm{kg}$ midazolam and anesthetized with $1 \mathrm{mcg} / \mathrm{kg}$ fentanyl (or $2 \mathrm{mg} / \mathrm{kg}$ ketamine).

The choice of CVC technique, either conventional (C-CVC) or ultrasoundguided CVC (USG-CVC), depended on equipment availability. The procedures were conducted by resident physicians specializing in pediatric intensive care supervised by an experienced preceptor or, occasionally, just by one experienced preceptor (or two, in the case of USG-CVC).

In $\mathrm{C}-\mathrm{CVC}$, the puncture site was determined by tactile and visual inspection of the anatomical reference sites, as described in the literature, and by using the Seldinger technique to pass the catheter; catheter location was then radiologically confirmed.

In USG-CVC, the site to be punctured was identified with the help of the portable Site-Rite Vision ${ }^{\circledast}$ Ultrasound System, whose transducer was covered with gel and, subsequently, with a sterile plastic protection while one of the operators (resident physician or preceptor) positioned the probe in a "short-axis" or "out-of-plane" approach. Once the vessel was identified on the ultrasound image, the depth could be determined by the markings on the right-hand side of the image. The other operator then performed the catheterization by the Seldinger technique; catheter location was then radiologically confirmed. 
Procedure time, number of attempts, and the outcome as to success rate and occasional complications were recorded.

\section{Results}

During the period from February 2016 to October 2016, 144 patients were admitted to the pediatric ICU referred for CVC with antibiotic therapy (117 patients), continuous sedation and hemodynamic monitoring (120 patients), administration of vasoactive drugs (48 patients) and other procedures such as hemodialysis, correction of hydroelectrolytic disorders and chemotherapy (8 patients). Sixty $60(41.7 \%)$ were females, age ranging from 10 days to 15 years (mean: 40.37 months; standard deviation: 54.31), with distribution frequencies by age group as summarized in Table 1 .

With respect to their nutritional status, $110(76.4 \%)$ of patients had a lower than normal BMI, $32(22.2 \%)$ had a normal BMI, and only $3(1.4 \%)$ had a condition compatible with overweight.

A total of 433 punctures were performed, with a mean of $3.01 \quad(s=2.66)$ punctures in 144 cases, success in 101 (70.41\%) of 144 cases, 34.03\% (49/144) of which cases were successful after a single puncture. CVC was USG-guided in 57 (39.58\%) of 144 cases. The time spent was, on average, $22.83(s=27.24)$ minutes.

With regard to the puncture sites, 94 of 144 procedures involved the jugular vein (65.3\%), 46 (31.9\%) involved the femoral vein, and 4 (2.8\%) involved the subclavian vein, with the right side having been predominant in the punctures (69.4\%), irrespective of the chosen vein.

Complications occurred in 32/144 cases (22.22\%), with arterial puncture being the most frequent one, 16/144 (11.11\%), followed by pneumothorax in $6 / 144$ (4.17\%), deep venous thrombosis in $2 / 144(1.39 \%)$, and other complications in $8 / 144(5.56 \%)$.

According to the value of the standard deviation relative to the means, age, time and number of attempts do not present normal distribution in the pediatric population who received treatment at the service. When considering the age groups found versus the frequencies of the variables puncture site, nutritional status and professional performing the procedure, the analysis of data

Table 1. Observed frequencies $(f \circ)$ of patients, and respective relative frequencies in percentage $(\%)$, for each age range.

\begin{tabular}{ccc}
\hline Age range & fo & $\%$ \\
\hline Neonate & 6 & 4.2 \\
Infant & 91 & 63.2 \\
Preschool & 16 & 11.1 \\
Child & 21 & 14.6 \\
Adolescent & 10 & 6.9 \\
Total & 144 & 100.0 \\
\hline
\end{tabular}


distributed in contingency tables would require a larger sample size due to the relatively rare categories.

Accordingly, for the purpose of analyzing any differences between the effects of CVC technique types on the outcomes, we used data from 94 of the 144 patients who, in a post hoc pairing, formed similar pairs for potentially intervening variables, especially differing as to the CVC technique type, composing two paired groups with 47 cases each. The levels of the potentially competing variables are shown in Table 2.

A comparison made between the USG-CVC and C-CVC groups as to the number of CVC attempts by using the Paired $t$-Test revealed a statistically significant difference $\left\{t=2.34, \mathrm{df}=46, t_{0.95}=2.02, p<0.05\right\}$. The number of attempts in the ultrasound-guided procedures was, on average, smaller (2.04) than the number of attempts when using the conventional technique (3.21).

A comparison made between the USG-CVC and C-CVC groups as to the time spent at CVC by using the Paired $t$-Test revealed a statistically significant difference $\left\{t=3.07, \mathrm{df}=46, t_{0,95}=2.02, p<0.05\right\}$. The mean time of the ultrasound-guided procedures was shorter (13.15 minutes) than the time spent on average when using the conventional technique (31.00 minutes).

The comparisons of the observed frequencies of success in catetherization and complications during this procedure between the paired groups (between techniques), considering the differences between pairs and within pairs, by means of ANOVA for dichotomized data, revealed that when two similar patients in terms of age group, nutritional status/BMI, are underwent to CVC at the same site by

Table 2. Potentially intervening variables and their respective levels, used in patient classification and ad hoc pairing, as well as their respective observed frequencies $(f 0)$ in both groups (CVC-USG and CVC-C) after the pairing.

\begin{tabular}{ccc}
\hline Intervening variables & Levels & fo \\
\hline Age & Neonate $(0-30$ days of age $)$ & 1 \\
& Infant $(1$ month - 2 years $)$ & 33 \\
Preschool $(2-6$ years $)$ & 6 \\
Nutritional status & Child $(6-12$ years $)$ & 2 \\
Adolescent $(12-18$ years $)$ & 39 \\
Puncture site & Low weight: BMI < 18.49 & 8 \\
Professional expertise & Normal: $18.49 \leq$ BMI $\leq 25.00$ & 0 \\
\hline
\end{tabular}

BMI, Body Mass Index; fo, observed frequency. 
professionals with equivalent expertise, ultrasound-guided catheterization resulted in less frequent complications, a statistically significant difference $\left(F_{(1,46)}=\right.$ 8.24; $Q_{(1)}=7.14, p<0.05$ ) (Table 3).

However, there was observed no statistically significant difference in the comparisons of success rates $\left(F_{(1,46)}=3.76 ; Q_{(1)}=3.56, p>0.05\right)$ (Table 4).

Success in the first CVC attempt occurred in $57.45 \%$ of cases (27/47) in the USG-CVC group versus 19.15\% (9/47) of cases in the C-CVC group. When the paired groups were compared, the success rate was greater in the elements of the pairs undergoing USG-CVC $\left(F_{(1,46)}=16.6 ; Q_{(1)}=12.5, p<0.05\right)$ (Table 5).

Table 3. ANOVA summary-table of comparison of differences among values of observed frequencies, at mean, of dependent variable "complications during CVC" considering the critical values for $F$ and Cochran $Q$ statistics as $F_{0.95}(1,46)=4.06$ and $\quad \chi_{0.95}^{2}(2-1)=3.84$, respectively, to verify the statistical significance at the adopted level.

\begin{tabular}{cccccc}
\hline & $\mathrm{SS}$ & $\mathrm{df}$ & $\mathrm{MS}$ & $F$ & $Q$ \\
\hline $\mathrm{SS}_{\text {between pairs }}$ & 6.28 & 46 & & & \\
$\mathrm{SS}_{\text {within pairs }}$ & 7.00 & 47 & & & \\
$\mathrm{SS}_{\text {techniques }}$ & 1.06 & 1 & 1.06 & 8.24 & 7.14 \\
$\mathrm{SS}_{\text {residual }}$ & 5.94 & 46 & 0.13 & & \\
$\mathrm{SS}_{\text {total }}$ & 13.28 & 93 & & & \\
\hline
\end{tabular}

SS, sum of squares; df, degrees of freedom; MS, mean of squares.

Table 4. ANOVA summary-table of comparison of differences among values of observed frequencies, at mean, of dependent variable "success in CVC" considering the critical values for $F$ and Cochran $Q$ statistics as $F_{0.95}(1,46)=4.06$ and $\chi_{0.95}^{2}(2-1)=3.84$, respectively, to verify the statistical significance at the adopted level.

\begin{tabular}{cccccc}
\hline & SS & df & MS & $F$ & $Q$ \\
\hline $\mathrm{SS}_{\text {between pairs }}$ & 9.81 & 46 & & & \\
$\mathrm{SS}_{\text {within pairs }}$ & 9.00 & 47 & & & \\
$\mathrm{SS}_{\text {techniques }}$ & 0.68 & 1 & 0.68 & 3.76 & \\
$\mathrm{SS}_{\text {residual }}$ & 8.32 & 46 & 0.18 & & \\
$\mathrm{SS}_{\text {total }}$ & 18.81 & 93 & & & \\
\hline
\end{tabular}

SS, sum of squares; df, degrees of freedom; MS, mean of squares.

Table 5. ANOVA summary-table of comparison of differences among values of observed frequencies, at mean, of dependent variable "Success in the first CVC attempt" considering the critical values for $F$ and Cochran $Q$ statistics as $F_{0.95}(1,46)=4.06$ and $\chi_{0.95}^{2}(2-1)=$ 3.84 , respectively, to verify the statistical significance at the adopted level.

\begin{tabular}{cccccc}
\hline & SS & df & MS & $F$ & $Q$ \\
\hline SS $_{\text {between pairs }}$ & 9.21 & 46 & & & \\
SS $_{\text {within pairs }}$ & 13.00 & 47 & & & \\
SS $_{\text {techniques }}$ & 3.45 & 1 & 3.45 & 16.60 & \\
SS $_{\text {residual }}$ & 9.55 & 46 & 0.21 & & \\
SS $_{\text {total }}$ & 22.21 & 93 & &
\end{tabular}

SS, sum of squares; df, degrees of freedom; MS, mean of squares. 


\section{Discussion}

The aim of this study was to investigate the effectiveness of USG during a CVC procedure in pediatric patients receiving care in an intensive care unit (ICU), where this technique is not part of the routine, due in part to the frequent unavailability of equipment, but still allowed for the constitution of a control group with the increasingly rare conventional technique. This method was found to be effective in this study, reducing the number of punctures, complications and time spent in the procedure.

Our results are in agreement with those reported in studies published in the literature in which, although no effect of the use of USG on the CVC success rate was found, most USG-guided procedures were successful and had fewer punctures, shorter times and a lower incidence of CVC-related complications. As expected, success after the first attempt was more frequent when the CVC procedure was USG-guided.

While the unpredictable availability of the USG equipment has prevented a matched-pairs design, the exposure of each patient to either one of the two techniques may have had some degree of randomness because of such unpredictability. On the other hand, the prospective nature of data acquisition may have, for the purposes of population description, a lower value than a retrospectively obtained sample, without the deliberate organization and precision in recording information. Nevertheless, descriptive parameters of the population distribution of the variables could be estimated and used in determining the sample size for future replications.

\section{Conclusion}

In this study, we showed that, in a sample of critical ICU patients, USG-guided $\mathrm{CVC}$ required fewer punctures, consumed less time, improved the overall success rate and facilitated the procedure in pediatric patients. USG-guided catheterization also minimized the occurrence of complications during the procedure, permitting clear visualization of the underlying anatomy and its variations. Therefore, we have recommended this technique be used in our pediatric intensive care unit, also for the training of new professionals.

\section{References}

[1] Okutubo, F.A. (1997) Central Venous Cannulation: How to Do It. British Journal of Hospital Medicine, 57, 368-370.

[2] Loeff, D.S., Matlak, M.E., Black, R.E., et al. (1982) Insertion of Small Central Venous Catheter in Neonates and Young Infants. Journal of Pediatric Surgery, 17, 944999. https://doi.org/10.1016/S0022-3468(82)80472-7

[3] Martin, C., Auffray, J.P., Saux, P., et al. (1986) The Axillary Vein: An Alternative Approach to Percutaneous Pulmonary Artery Catheterization. Chest, 90, 694-697. https://doi.org/10.1378/chest.90.5.694

[4] Ruesch, S., Walder, B.W. and Tramèr, M.R. (2002) Complications of Central Venous Catheters: Internal Jugular versus Subclavian Access-A Systematic Review. 
Critical Care Medicine, 30, 454-460.

https://doi.org/10.1097/00003246-200202000-00031

[5] Haire, W.D. and Lieberman, R.P. (1994) Defining the Risks of Subclavian-Vein Catheterization. The New England Journal of Medicine, 331, 1769-1770.

https://doi.org/10.1056/NEJM199412293312609

[6] Paiva, B.S., Fioretto, J.R., Paiva, C.E., et al. (2006) Cateterização Venosa Central em Crianças Internadas em Unidade de Terapia Intensiva Pediátrica: Complicações. Revista Paulista de Pediatria, 24, 35-41.

[7] Ullman, J.I. and Stoelting, R.K. (1978) Internal Jugular Vein Location with the Ultrasound Doppler Blood Flow Detector. Anesthesia \& Analgesia, 57, 118. https://doi.org/10.1213/00000539-197801000-00024

[8] Lichtenstein, D.A. (2004) General Ultrasound in the Critically Ill. Springer-Verlag, Berlin.

[9] Beaulieu, Y. and Marik, P.E. (2005) Bedside Ultrasonography in the ICU: part 2. Chest, 128, 1766-1781. https://doi.org/10.1378/chest.128.3.1766

[10] Hind, D., Calvert, N., McWilliams, R., et al. (2003) Ultrasonic Locating Devices for Central Venous Cannulation: Meta-Analysis. BMJ, 327, 361.

https://doi.org/10.1136/bmj.327.7411.361

[11] US Agency for Healthcare Research and Quality (2001) University of California San Francisco-Stanford Evidence-Based Practice Center: Making Health Care Safer: A Critical Analysis of Patient Safety Practices. Evidence Report/Technology Assessment No. 43. Agency for Healthcare Research and Quality, US Department of Health and Human Services, Rockville.

https://archive.ahrq.gov/clinic/ptsafety/pdf/ptsafety.pdf

[12] National Institute for Health and Care Excellence (2002) Guidance on the Use of Ultrasound Locating Devices for Placing Central Venous Catheters. Technology Appraisal Guidance; No. 49. National Institute for Health and Care Excellence, London.

[13] Shekelle, P.G. and Dallas, P. (2013) Use of Real-Time Ultrasound Guidance during Central Line Insertion: Brief Update Review. Evidence Report/Technology Assessment Number 211-Making Health Care Safer II: An Updated Critical Analysis of the Evidence for Patient Safety Practices. Agency for Healthcare Research and Quality-US Department of Health and Human Services, Rockville. https://archive.ahrq.gov/research/findings/evidence-based-reports/ptsafetyII-full.pdf

[14] Miller, A.H., Roth, B.A., Mills, T.J., et al. (2002) Ultrasound guidance versus the landmark technique for the placement of central venous catheters in the emergency department. Academic Emergency Medicine, 9, 800-805. https://doi.org/10.1111/j.1553-2712.2002.tb02168.x

[15] Denys, B.G. and Urestsky, B.F. (1991) Anatomical Variation of the Internal Jugular Vein Location: Impact on Central Venous Access. Critical Care Medicine, 19, 15161519. https://doi.org/10.1097/00003246-199112000-00013

[16] Gordon, A.C., Saliken, J.C., Johns, D., et al. (1998) US-Guided Puncture of the Internal Jugular Vein: Complications and Anatomic Considerations. Journal of Vascular and Interventional Radiology, 9, 333-338. https://doi.org/10.1016/S1051-0443(98)70277-5

[17] Legler, D. and Nugent, M. (1984) Doppler Localization of the Internal Jugular Vein Facilitates Central Venous Cannulation. Anesthesiology, 60, 481-482. https://doi.org/10.1097/00000542-198405000-00016

[18] Cobb, L.M., Vinocur, C.D., Wagner, C.W., et al. (1987) The Central Venous Anat- 
omy in Infants. Surgery, Gynecology \& Obstetrics, 165, 230-234.

[19] Steinberg, C., Weinstock, D.J., Gold, J.P., et al. (1992) Measurements of Central Blood Vessels in Infants and Children: Normal Values. Catheterization and Cardiovascular Diagnosis, 27, 197-201. https://doi.org/10.1002/ccd.1810270308

[20] Alderson, P.J., Burrows, F.A., Stemp, L.I., et al. (1993) Use of Ultrasound to Evaluate Internal Jugular Vein Anatomy and to Facilitate Central Venous Cannulation in Paediatric Patients. British Journal of Anaesthesia, 70, 145-148. https://doi.org/10.1093/bja/70.2.145

[21] Troianos, C.A., Kuwik, R.J., Pasqual, J.R., et al. (1996) Internal Jugular Vein and Carotid Artery Anatomic Relation as Determined by Ultrasonography. Anesthesiology, 85, 43-48. https://doi.org/10.1097/00000542-199607000-00007

[22] Souza Neto, E.P., Grousson, S., Duflo, F., et al. (2014) Ultrasonographic Anatomic Variations of the Major Veins in Paediatric Patients. British Journal of Anaesthesia, 112, 879-884. https://doi.org/10.1093/bja/aet482

[23] Verghese, S.T., McGill, W.A., Patel, R.I., et al. (2000) Comparison of Three Techniques for Internal Jugular Vein Cannulation in Infants. Pediatric Anesthesia, 10, 505-511. https://doi.org/10.1046/j.1460-9592.2000.00554.x

[24] Verghese, S.T., McGill, W.A., Patel, R.I., et al. (1999) Ultrasound-Guided Internal Jugular Venous Cannulation in Infants: A Prospective Comparison with the Traditional Palpation Method. Anesthesiology, 91, 71-77. https://doi.org/10.1097/00000542-199907000-00013

[25] Froelich, C.D., Rigby, M.R., Rosenberg, E.S., et al. (2009) Ultrasound-Guided Central Venous Catheter Placement Decreases Complications and Decreases Placement Attempts Compared with the Landmark Technique in Patients in a Pediatric Intensive Care Unit. Critical Care Medicine, 37, 1090-1096. https://doi.org/10.1097/CCM.0b013e31819b570e

[26] Jijeh, A.M., Shaath, G., Kabbani, M.S., et al. (2014) Ultrasound Guided Vascular Access in Pediatric Cardiac Critical Care. Journal of the Saudi Heart Association, 26, 199-203. https://doi.org/10.1016/j.jsha.2014.04.003

[27] Pirotte, T. and Veyckemans, F. (2007) Ultrasound-Guided Subclavian Vein Cannulation in Infants and Children: A Novel Approach. British Journal of Anaesthesia, 98, 509-514. https://doi.org/10.1093/bja/aem041

[28] Pérez-Quevedo, O., López-Álvarez, J.M., Limiñana-Cañal, J.M., et al. (2016) Design and Application of Model for Training Ultrasound-Guided Vascular Cannulation in Pediatric Patients. Medicina Intensiva, 40, 364-360. https://doi.org/10.1016/j.medin.2015.11.005

[29] Grebenik, C.R., Boyce, A., Sinclair, M.E., et al. (2004) NICE Guidelines for Central Venous Catheterization in Children. Is the Evidence Base Sufficient? British Journal of Anaesthesia, 92, 827-830. https://doi.org/10.1093/bja/aeh134

[30] Iwashima, S., Ishikawa, T. and Ohzeki, T. (2008) Ultrassound-Guided Versus Landmark-Guided Femoral Vein Access in Pediatric Cardiac Catheterization. Pediatric Cardiology, 29, 339-342. https://doi.org/10.1007/s00246-007-9066-2 
Submit or recommend next manuscript to SCIRP and we will provide best service for you:

Accepting pre-submission inquiries through Email, Facebook, LinkedIn, Twitter, etc. A wide selection of journals (inclusive of 9 subjects, more than 200 journals)

Providing 24-hour high-quality service

User-friendly online submission system

Fair and swift peer-review system

Efficient typesetting and proofreading procedure

Display of the result of downloads and visits, as well as the number of cited articles Maximum dissemination of your research work

Submit your manuscript at: http://papersubmission.scirp.org/

Or contact ojped@scirp.org 